\title{
Análise preliminar das manifestações sonoras e do habitat de Picumnus nebulosus Sundevall (Aves, Picidae)
}

\author{
Luiz dos Anjos 1
}

\begin{abstract}
Preliminary analysis of sounds and habitat of Picumnus nebulosus Sundevall (Aves, Picidae). Observations and recordings of Picumnus nebulosus Sundevall, 1866 were carried out at Santa Rita farm ( $21^{\circ} 15^{\prime}$ 'S, $\left.50^{\circ} 00^{\prime}\right)$, municipality of Palmeira, state of Paraná, southern Brazil, from 1984 to 1991. The song of this species $(0.25 \mathrm{~s}$ to $0.64 \mathrm{~s}$ total time) is composed by a series of four to six high pitched ( 6.3 to $7.8 \mathrm{kHz})$ and short $(0.03$ to $0.05 \mathrm{~s})$ notes $(n=23)$. The fight call is a series of very short notes emitted between 2 to $8 \mathrm{kHz}$ during $0.23 \mathrm{~s}$ to $0.32 \mathrm{~s}(\mathrm{n}=34)$. The drum, a sequence of pulses, is emitted during 3.5 to $7.2 \mathrm{~s}(\mathrm{n}=42)$; the four sequences in the begining are composed by four pulses and the rest by three pulses. P. nebulosus was more abundant in forest patches than in continuous forest. It was usually watched foraging in branches 1 to $4 \mathrm{~mm}$ diameter at mid-level; foraging in bamboo, the most common habitat cited in the literature, was registered in less than $10 \%$ of the observations.
\end{abstract}

KEY WORDS. Picumnus nebulosus, sounds, habitat, abundance, araucaria forest

No grupo dos pica-paus anões, Picumnus nebulosus Sundevall, 1866 (Picidae) é provavelmente a espécie de distribuição mais austral na América, atingindo o sul do Uruguai, além de incluir os estados de São Paulo, Paraná, Santa Catarina e Rio Grande do Sul, no Brasil, e o Departamento de Missiones, na Argentina (PINTO 1978; SHORT 1982; Belton 1984; CUEllo 1985; SiCK 1985, 1997; NAROSKY \& YZURIETA 1987; SCHERER-NETO \& STRAUBE 1995). Esta espécie foi considerada por CRACRAFT (1985) como um taxa representativo de um suposto centro evolutivo Paraná, que estaria associado à ocorrência da floresta de araucária (floresta ombrófila mista), comum no sul do Brasil. Como outras espécies do mesmo gênero, são poucas as informações relativas aos seus hábitos, as quais se encontram incluídas em publicações de abordagem geral (SHORT 1982; BELTON 1984; SiCK 1985, 1997; FRUGIS et al. 1988; WINKLER et al. 1995).

Em relação às manifestações sonoras, os pica-paus são conhecidos por produzir sons rítmicos enquanto bicam troncos e galhos de árvores e outros tipos vegetais (tamborilar). Este som tem sido associado à proclamação territorial. O ritmo de batidas na madeira é nitidamente diferente daquele produzido enquanto a ave forrageia (SHORT 1982; WinKLER et al. 1995). Além do tamborilar, os pica-paus apresentam um repertório vocal no qual pode ser reconhecido o canto, com função

1) Departamento de Biologia Animal e Vegetal, Universidade Estadual de Londrina. Caixa Postal 6001, 86051-970 Londrina, Paraná, Brasil.

E-mail: llanjos@sercomtel.com.br 
similar ao tamborilar, ambos associados ao reconhecimento especifico (SHORT 1982; SiCK 1985, 1997; VIELLIARD 1986; WINKLER et al. 1995).

O objetivo deste estudo é apresentar observações de campo de $P$. nebulosus e analisar, de forma preliminar, as manifestações sonoras e a ocupação do habitat. São utilizadas informações sobre a abundância da espécie em floresta de araucária obtidas durante um levantamento quantitativo de aves.

\section{MATERIAL E MÉTODOS}

\section{Local de estudo}

As observações e gravações foram desenvolvidas na fazenda Santa Rita $\left(21^{\circ} 15^{\prime} \mathrm{S}, 50^{\circ} 00^{\prime} \mathrm{W}\right)$, município de Palmeira, Paraná, em uma área estimada de 2000 ha. Aproximadamente metade desta área apresenta uma fitofisionomia constituída por campo, onde existem, dispersos entre si, capões de floresta de araucária, com 0,5 ha a 100 ha de superfície; na porção restante existe uma floresta de araucária contínua. As espécies arbóreas Araucaria angustifolia Bert. O. Kuntze (Araucariaceae), Schinus terebenthifolius Raddi (Anacardiaceae), Eugenia uniflora Linnaeus (Myrtaceae), Campomanesia xantocarpha Berg (Myrtaceae), Piptocarpha angustifolia Dusen (Asteraceae), Nectandra lanceolata (Nees \& Martius) (Lauraceae) e Cedrella fissilis Velloso (Meliaceae) são as mais abundantes na floresta de araucária da região, tanto na área contínua como nos capões. No campo predomina Axonopus brasiliensis (Sprengel) (Poaceae). Uma descrição mais detalhada da vegetação desta região é apresentada em KLEIN \& HATSCHBACH (1970). A temperatura média anual está entre $12,1^{\circ} \mathrm{C}$ e $24,3^{\circ} \mathrm{C}$ (MAACK 1981 ).

\section{Métodos}

A espécie P. nebulosus foi registrada na fazenda Santa Rita (ANJOS \& GRAF 1993) tendo sido observada no período de 1984 a 1991 em várias ocasiões. As manifestações sonoras foram gravadas em gravador de rolo UHER 4400 a uma velocidade de $19 \mathrm{~cm} / \mathrm{s}$ com a utilização de microfone unidirecional e refletor parabólico. As gravações estão depositadas no Laboratório de Ornitologia da Universidade Estadual de Londrina com números de referência precedidos pelo código LDA. A análise dos sons foi desenvolvida no programa Avisoft Sonograph Pro para Windows 3.11.

De fevereiro de 1990 a janeiro de 1991 foi aplicado o método de amostragem por pontos de escuta, conforme BLONDEL et al. (1970), na área contínua de floresta de araucária de 1000 ha e em dois capões com 9 ha e 20 ha. Através desta metodologia se obteve uma medida da abundância da espécie nestes três locais, que é apresentada na forma de índice pontual (número de contatos com a espécie em pontos pré-determinados dividido pelo total de amostras). $O$ capão de 20 ha está a 900 metros da área contínua, mas encontra-se ligado a ela através de uma estreita mata ciliar. O capão de 9 ha está totalmente isolado e a 2000 metros da área contínua de floresta de araucária. 


\section{RESULTADOS E DISCUSSÃO}

\section{Manifestações sonoras}

\section{Canto e "Canto de Briga"}

Em 15/6/86 (8:30 h) foi gravada uma seqüência de notas de som agudo de um indivíduo que as emitia em intervalos de forrageamento (LDA 10/1). O indivíduo percorria o estrato inferior ( 1 a 2,5 m) da borda do capão de 20 ha, pousava freqüentemente em exemplares jovens do bambu Merostachys multiramea (Poaceae), além de arbustos e arvoretas. A ave reagiu rapidamente ao playback desta gravação com aproximação, mas não vocalizou. Em diversas oportunidades registramos a presença de $P$. nebulosus através desta vocalização que pode ser ouvida a pelo menos 50 metros de distância, sendo provavelmente a mais comumente emitida pela espécie.

Em 1/9/90 (10:30 h) apresentamos a gravação LDA 10/1 a um indivíduo que forrageava no interior de outro capão de floresta de araucária ( 9 ha) na mesma fazenda. Este indivíduo percorria o estrato médio (entre 5 e 7 metros de altura) pousando normalmente em galhos de árvores com 10 a 12 metros de altura. $\mathrm{O}$ diâmetro dos galhos percorridos pela espécie era de $1 \mathrm{~cm}$ a $4 \mathrm{~cm}$. A ave reagiu fortemente ao playback com aproximação e com uma vocalização, em forma de trinado e de baixa propagação no ambiente, que foi gravada (LDA 75/12).

A gravação LDA 10/1 corresponde ao canto da espécie, conforme VIELLIARD (1986), em função da forte reação ao playback e pela grande propagação que atinge no ambiente, conforme VIELLIARD (1986), características importantes no reconhecimento específico durante a proclamação territorial. Esta vocalização é constituída por 4 a 6 notas melódicas, de som agudo, emitidas entre 7,8 e $6,3 \mathrm{kHz}(\mathrm{n}=23)$ (Fig. 1). A primeira nota, sempre presente, apresenta uma modulação ascendente sendo emitida em uma freqüência entre 7,1 e $7,8 \mathrm{kHz}$. As notas seguintes, em número de 3 a 5 , têm forma de arco (modulação ascendente e descendente) sendo emitidas entre 6,3 e 7,4 kHz (Fig. 4). A primeira nota é nitidamente mais curta (média de $0,03 \mathrm{~s}$ ) que as outras (médias entre 0,04 e $0,05 \mathrm{~s}$ ). A terceira $(0,04 \mathrm{~s})$ e quarta $(0,05 \mathrm{~s})$ notas foram geralmente as mais longas. $O$ intervalo entre as notas é de 0,03 a $0,10 \mathrm{~s}$ e a duração total do canto, variando em função do número de notas, está entre 0,25 (4 notas) e $0,64 \mathrm{~s}$ (6 notas). Esta vocalização corresponde provavelmente ao zunido descrito por SICK $(1985,1997)$ e por WINKLER et al. (1995). No Rio Grande do Sul BELTON (1984) a descreveu como uma seqüência de notas curtas e agudas pouco espaçadas.

A gravação LDA 75/12 corresponde ao "canto de briga" por ser emitido como resposta ao playback do canto em situação de defesa territorial, como definido por VIELLIARD (1986). Esta vocalização é composta por quatro a seis notas de curta duração e súbita variação de freqüência (pseudopulsos), emitidas em rápida seqüência produzindo um som trinado $(n=34)$ (Fig. 2). A freqüência está entre 2 e $8 \mathrm{kHz}$ sendo que a energia é concentrada acima de $7 \mathrm{kHz}$. O tempo de duração do trinado está entre 0,23 e $0,32 \mathrm{~s}$. 

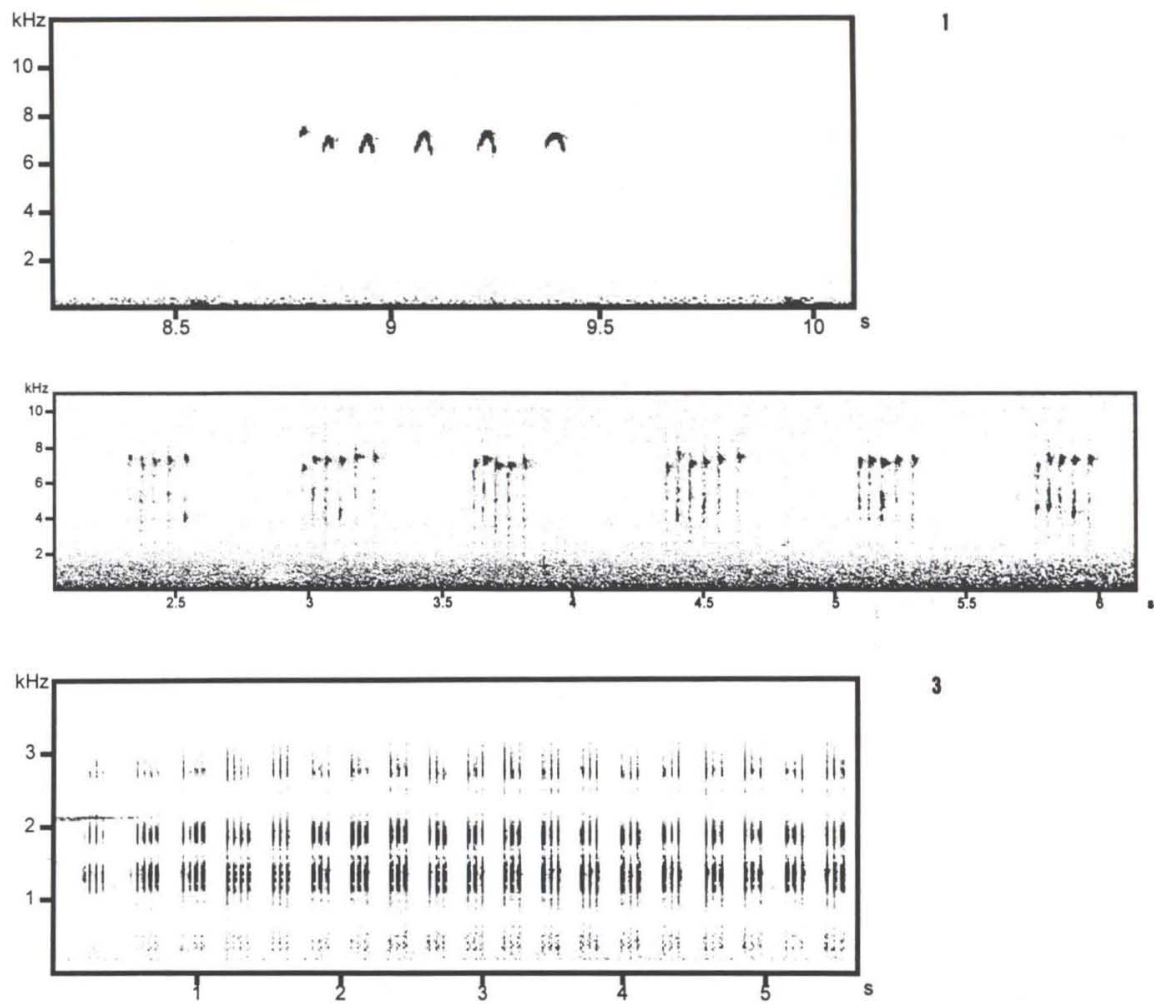

Figs 1-3. Sonogramas das manifestações sonoras de Picumnus nebulosus (obtidas através do programa Avisoft Sonograph Pro). (1) canto (LDA 10/1); (2) "canto de briga" (LDA 75/12); (3) tamborilar (LDA 76/10). Todas as gravações realizadas na fazenda Santa Rita, Palmeira, Paraná.

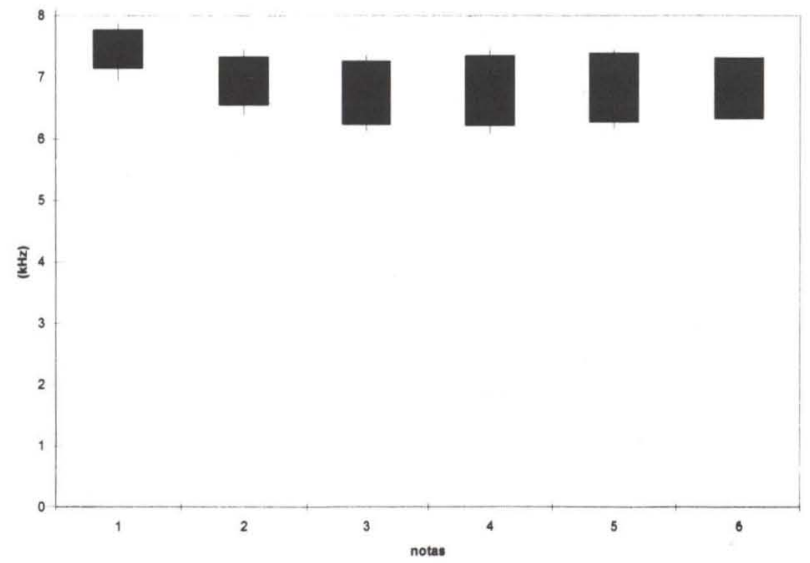

Fig 4. Espectro de freqüência (em kHz) das notas do canto de Picumnus nebulosus $(\mathrm{n}=23)$. 


\section{Tamborilar}

Em 4/9/90 (6:45 h) foi gravado o som rítmico do tamborilar (LDA 76/10), que podia ser ouvido a uma distância superior a 50 metros; durante quinze minutos a ave permaneceu no mesmo ponto produzindo este som quando bicava um galho de $4 \mathrm{~cm}$ de diâmetro de árvore de médio porte (20 metros de altura) a aproximadamente $5 \mathrm{~m}$ do solo, no interior do capão de 9 ha. Ao playback do tamborilar o indivíduo reagiu com aproximação e vocalização (o "canto de briga" descrito anteriormente).

Em 5/6/91 (11:20 h) foi observado um indivíduo forrageando no estrato médio de interior do mesmo capão. $\mathrm{O}$ indivíduo pousava freqüentemente em galhos de até $4 \mathrm{~cm}$ de diâmetro. Bicava com vigor a madeira sendo o barulho (LDA 91/3), não rítmico, ouvido a pelo menos 30 metros de distância. Não houve reação do indivíduo ao playback desta manifestação sonora.

O tamborilar analisado em sonograma é constituído por uma longa seqüência (frase) de grupamentos de notas (estrofe) em forma de pulsos (Fig 3). Em todas as frases analisadas em sonograma $(n=42)$, as quatro primeiras estrofes foram constituídas por quatro notas, sendo as seguintes constituídas por três notas. A freqüência de emissão cobre uma faixa de até $3 \mathrm{kHz}$, sendo que a energia está concentrada entre 1 e $2 \mathrm{kHz}$. O número de estrofes em cada frase variou de 12 a 23 . A duração total de cada frase está entre 3,5 e 7,2 s e o de cada estrofe entre 0,14 e $0,26 \mathrm{~s}$. O intervalo entre as estrofes é em média de $0,15 \mathrm{~s}$. O som produzido durante o forrageamento é contituído por notas em forma de pulsos emitidas isoladamente em intervalos não padronizados.

BELTON (1984) e SICK $(1985,1997)$ haviam aparentemente registrado o tamborilar apenas quando a ave estava pousada em bambu. BELTON (1984) menciona que o tamborilar é constituído por duas, três ou quatro batidas (notas). Em todas as gravações analisadas no presente estudo o tamborilar apresentou um padrão: as quatro primeiras estrofes sempre foram constituídas por quatro notas e as seguintes por três.

\section{Habitat}

Os valores do índice pontual de abundância de $P$. nebulosus foram significativamente diferentes (teste de Qui-quadrado $\mathrm{p}<0,05$ ): 0,014 na área contínua de floresta de 1000 ha; 0,042 no capão de 20 ha e 0,292 no capão de 9 ha. Estes valores indicam um aumento da abundância quanto menor for a área de floresta.

$\mathrm{O}$ habitat de $P$. nebulosus tem sido relacionado à borda da floresta, sendo especialmente comum em áreas de bambu (SHORT 1982; BELTON 1985; WINCKLER et al. 1995; SicK 1985, 1997). Na fazenda Santa Rita esta espécie ocorreu tanto no interior da floresta como em capões. Entretanto, a abundância foi nitidamente superior nos capões, inclusive aumentando com a dimunição da área e com o isolamento; supostamente a espécie é favorecida pelo aumento da área de borda nos capões menores. Em capões o bambu não é muito comum, o que sugere que $P$. nebulosus não está tão intimamente relacionado a este tipo vegetal. Apenas em cinco observações, dentre o total de 56 ao longo do período de estudo na fazenda Santa Rita, esta espécie foi registrada em bambu. 
AGRADECIMENTOS. Agradeço ao senhor José Carlos Veiga Lopes pelo incentivo e apoio aos trabalhos de campo em sua propriedade, a fazenda Santa Rita. Em quase todas as amostragens de campo tivemos a companhia de Roberto Boçon, a quem agradecemos sinceramente. Agradeço também a Jacques M. E. Vielliard, pela revisão do manuscrito, e à Marilda Carvalho Dias e Alba Lúcia Cavalheiro, pelo auxílio na descrição da vegetação. Parte da pesquisa bibliográfica e a análise sonográfica foi desenvolvida em junho/julho de 1997 no Museu de Zoologia e Instituto de Pesquisa em Zoologia Alexander Koenig (Bonn, Alemanha) (agradeço especialmente à Christa Budde pelo apoio técnico); apoio financeiro para viagem e estadia nesta Instituição alemã, para pesquisa com Karl-Ludwig Schuchmann, foi fornecido pelo Convênio de Cooperação Internacional Brasil/Alemanha CNPq/DAAD. Suporte financeiro para trabalhos de campo na bacia do rio Tibagi tem sido obtido através do convênio Universidade Estadual de Londrina/Consórcio Intermunicipal para Preservação Ambiental do Rio Tibagi/Klabin Fabricadora de Papel e Celulose. Apoio para trabalhos sobre a avifauna em fragmentos florestais tem sido recebido do CNPq na forma de Bolsa Produtividade em Pesquisa (processo 520057/95-3).

\section{REFERÊNCIAS BIBLIOGRÁFICAS}

ANJOS, L. DOS \& V. GRAF. 1993. Riqueza de aves da fazenda Santa Rita, região dos Campos Gerais, Palmeira, Paraná, Brasil. Revta bras. Zool. 10 (4): 673-693.

Belton, W. 1984. Birds of Rio Grande do Sul, Brazil, Part 1. Bull. Amer. Mus. Nat. Hist. 178: 369-636.

Blondel, J.; C. FERRY \& B. Frochot. 1970. La méthode des indices ponctuels d'abondance (I.P.A.) ou des relevés d'avifaune par "stations d'écoute". Alauda 38: $55-71$.

CRACRAFT, J. 1985. Historical biogeography and patterns of diferentiation within the South America avifauna: Areas of endemism. Ornith. Monogr. 36: 49-84.

CUEllo, J.P. 1985. Lista de referencia y bibliografia de las aves uruguayas. Montevideo, Museo Damaso Antonio Larranaga 1, 116p.

Frugis, S.; G. MalaguzZi; G. ViCinI \& P. CRIstina. 1988. Guida ai picchi del mondo. Torino, Museo Regionale di Scienze Naturali, 345p.

KLEIN, R.M. \& G. HATSChBACH. 1970. Fitofisionomia e notas complementares sobre o mapa fitogeográfico de Quero-quero (Paraná). Bol. Par. Geoc. 28 (29): 159-188.

MAACK, R. 1981. Geografia Física do Estado do Paraná. Rio de Janeiro, Livraria José Olympio, 450p.

NAROSKY, T. \& D. YZURIETA. 1987. Guia para la identificacion de las aves de Argentina y Uruguay. Buenos Aires, Vazquez Mazzini, 340p.

PinTo, O.M.O. 1978. Novo catálogo das aves do Brasil. São Paulo, Empresa Gráfica das Rev. dos Tribunais, Vol. 1, 446p.

Scherer-Neto, P. \& F. C. Straube. 1995. Aves do Paraná. História, lista anotada e bibliografia. Curitiba, Ed. dos autores, 79p.

SHORT, L. L. 1982. Woodpeckers of the world. Delaware, Delaware Museum of Natural History, Monograph Series Number 4, 676p.

Sick, H. 1985. Ornitologia brasileira, uma introdução. Brasília, Ed. Universidade de Brasília, 827p. 
1997. Ornitologia brasileira. Rio de Janeiro, Ed. Nova Fronteira, 862p. VIELLIARD, J.M.E. 1986. O uso da Bioacústica na Observação de Aves, p. 98-121. In: Anais do II Encontro Nacional de Anilhadores de Aves. Rio de Janeiro, Ed. Universidade Federal do Rio de Janeiro, 230p.

WINKLER, H.; D.A. CHRISTIE \& D. NUMEY. 1995. Woodpeckers: a guide to the woodpeckers of the world. New York, Houghton Mifflin Company, 406p.

Recebido em 31.X.1997; aceito em 31.III.1999. 